OLE MICHAEL SELBERG

\title{
The Lectureship in Norwegian at the Adam Mickiewicz University, Poznań
}

The author has been asked to furnish this periodical with some information about the above lectureship, which was established in 1960 in accordance with the Cultural Agreement between the People's Republic of Poland and the Kingdom of Norway, and which is associated with the A. M. University, Poznań. To begin with, the position as a lecturer was held by Mr Erik Baugeröd, who remained lecturer until Christmas 1962 when he was replaced by $\mathrm{Mr}$ Folke Christensen. From the fall $1964 \mathrm{Mr}$ Ole Michael Selberg is lecturer. There exist no detailed instructions concerning the lectureship on Norwegian side but the Norwegian Ministry of Foreign Affairs expects the lecturer to carry on information work about Norway. On Polish side the lecturer is obliged to give lectures in Norwegian under the supervision of Prof. Dr L. Zabrocki, head of the Department of Germanic Languages. All students in this department are required to take at least two years (two hrs a week) of a Scandinavian language. Although in principle the students are free to choose between Danish, Norwegian and Swedish there is at the moment no Danish or Swedish lectureship at the University, corresponding to the Norwegian one. However one of the Polish colleagues conducts classes in Swedish on the elementary and advanced level and efforts are being made to establish a Danish lectureship. - The Norwegian lecturer's contract with the A. M. University expires on the 31st of August every year.

Unfortunately I am not very well acquainted with the activities of my predecessors but I think one of the most serious difficulties for all three of us has been the lack of adequate textbooks. The existing grammars in German and English are all rather unsatisfactory for courses of this kind and so the lecturer is to a great extent thrown back on his own resources and his own ingenuity. I have the impression that after a period of experimentation my predecessors have chosen to write most of the texts themselves - an approach which seems to have given comparatively good results, judging from the standard of the advanced students. As for myself I have during my first semester tentatively used a new textbook, Elementary Norwegian by Berulfsen and Boardman, eking it out with texts written by myself whenever I considered it necessary. In spite of its many merits the book is not ideal for our ends (this is chiefly because it is written with an intensive course in mind, alternating between classroom instruction and laboratory sessions) but with a little adaptation I have found it very useful. Since for various reasons, independent on the University or myself, I was able to start teaching only at the beginning of November 1964 it is still rather early to make any statements about the progress of the students. With the low 
number of classes per week a realistic appreciation of the results one can expect to achieve is necessary; I think one should primarily strive to give the students a certain oral command of the language, based on a small, active vocabulary and a proficiency in the use of the simpler syntactical patterns. At the end of the second year the students should also be able to find their way through a modern Norwegian text by the aid of a dictionary. In accordance with this the classes are at the beginning conducted as intensive conversation exercises, the syntactical patterns being introduced successively, according to their degree of complexity. Later readings are introduced, dealing with various aspects of Norwegian culture and background. Acquiring a native pronunciation cannot in my opinion be the main goal of the instruction. Too much drilling of foreign sounds during language classes for adults only give meagre results in most cases and sometimes is a mere waste of time. A certain insistence on pronunciation is however necessary if the students are to learn to make themselves understood.

Fortunately students who are especially interested in Scandinavian philology now can obtain scholarships for one year's study at the University of Oslo. At the moment there are two students and one assistant from the A. M. University benefiting from such scholarships. In addition there exist scholarships to the International Summer School at the University of Oslo, which offers intensive language courses. The scholarships are in my opinion especially valuable because of the haphazard character of the Scandinavian library at the A. M. University which makes it extremely difficult to do any work on Scandinavian philology here in Poznań. It is to be hoped that this situation will soon be remedied. 